\title{
Combining Registration and Minimum Surfaces for the Segmentation of the Left Ventricle in Cardiac Cine MR Images
}

\author{
Marie-Pierre Jolly, Hui Xue, Leo Grady, and Jens Guehring \\ Siemens Corporate Research, Imaging and Visualization Department Princeton, NJ \\ marie-pierre.jolly@siemens.com
}

\begin{abstract}
This paper describes a system to automatically segment the left ventricle in all slices and all phases of cardiac cine magnetic resonance datasets. After localizing the left ventricle blood pool using motion, thresholding and clustering, slices are segmented sequentially. For each slice, deformable registration is used to align all the phases, candidates contours are recovered in the average image using shortest paths, and a minimal surface is built to generate the final contours. The advantage of our method is that the resulting contours follow the edges in each phase and are consistent over time. We demonstrate using 19 patient examples that the results are very good. The RMS distance between ground truth and our segmentation is only 1.6 pixels $(2.7 \mathrm{~mm})$ and the Dice coefficient is 0.89 .
\end{abstract}

\section{Introduction}

Cardiovascular disease is now the largest cause of death in the modern world and is an important health concern. Physicians use non invasive technologies such as magnetic resonance (MR) imaging to observe the behavior of the heart and more specifically the left ventricle (LV). They want to quantify important measures such as blood pool volume over time, myocardial mass, ejection fraction, cardiac output, peak ejection rate, filling rate, myocardial thickening, which can all be computed with an outline of the LV. Manual outlining in all images is very cumbersome however and most physicians limit it to the end-diastolic (ED) and end-systolic (ES) phases, which is enough to calculate ejection fraction, but not enough to estimate some of the other quantities. This paper proposes a system to automatically segment the LV in all slices and all phases of a cardiac MR cine study.

MR cine consists of $4 \mathrm{D}(3 \mathrm{D}+\mathrm{T})$ data and the segmentation of all the images can be tackled in various ways. Some researchers have attempted 4D segmentation [1. We believe however that this approach is not feasible, since it is very difficult to build a model that is general enough to cover all possible shapes and dynamics of the LV and a model-free approach would not be constrained enough. The opposite approach of segmenting each image individually [2] results in little cohesion between images and unsmooth contours over time. The intermediate approach used very often of segmenting the LV in one phase on all slices [34] 


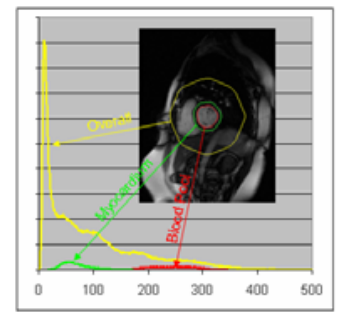

(a)

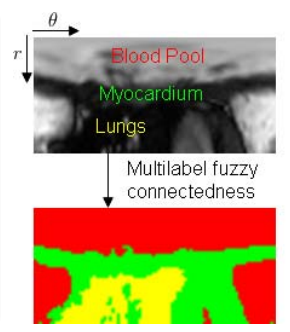

(b)

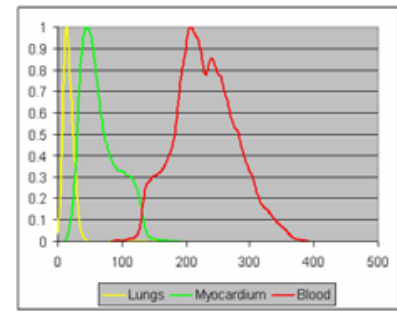

(c)

Fig. 1. Gray level analysis: (a) Original histograms; (b) Polar image and multiseeded fuzzy connectedness region labeling; (c) Final histograms

can be quite difficult. When a model is used, it needs to be carefully trained for all possible LV shapes and all possible MR acquisition protocols. Conversely, image-base techniques tend to be ad-hoc. We have chosen instead to segment all phases in one slice and propagate the segmentation between slices. This method can take advantage of the strong temporal correlation between phases to segment individual slices. For temporal propagation, researchers have proposed using a dynamic model of the LV [5,6] while other methods incorporate a tracking component into the recovery for process 78 . We use deformable registration to align all phases and generate an average image for segmentation. We use minimal surfaces to enforce consistency between phases so that contours follow image edges in each phase and are smooth over time.

We will describe the different steps of the algorithm and demonstrate on 19 patient datasets that the segmented contours are very close to manually defined ground truth contours.

\section{Left Ventricle Segmentation}

The proposed algorithm is divided into the following steps: 1) Heart and left ventricle blood pool detection; 2) Polar space transformation; 3) Gray scale analysis; 4) Segmentation of the first slice, which comprises of deformable registration to align all the phases, segmentation of the average temporal image and minimal surface segmentation of all phases; and 5) Segmentation of the other slices. We will describe each of these steps in more details in the next sections.

\subsection{Heart and Left Ventricle Blood Pool Detection}

For the detection of the heart and the LV blood pool, we use the method proposed by Jolly [9. It uses the first harmonic of the Fourier transform in each slice to detect the beating heart. Then, blood-like connected components are extracted using Otsu thresholding and characterized by their shape, temporal behavior, position, etc. Finally, isoperimetric clustering is used to group connected components between slices and form the LV blood pool. This process 
does not generate a blood pool region on all slices, nor does it handle the papillary muscles correctly in the blood pool region, but it is a good starting point for the rest of our algorithm.

\subsection{Polar Space Transformation}

We have chosen to work in polar space for multiple reasons. First, the contours to be recovered are roughly circular. Second, the segmentation will be performed using a shortest path algorithm which is well known to be biased toward small contours in Cartesian space. In polar space however, all contours have the same size since they start on one side of the image and end on the other, so there is no bias on the length of the contour. Finally, the images in Cartesian space are around $256 \times 256$ while in polar space, they are around $50 \times 90$ making the processing much faster. The center and maximum radius of the polar space are calculated from the blood pool estimates.

\subsection{Gray Level Analysis}

Because no two MR acquisitions are the same, it is important to determine the gray level properties of the images in the current dataset. We use the multiseeded fuzzy connectedness approach proposed in [10] to build histograms for the lungs, myocardium and blood pool distributions. The process is illustrated in Fig. 1 . Approximate histograms are built using the blood pool regions recovered in Section 2.1, the pixels in a small ring around those regions, and a larger region (largely consisting of lung pixels). The pixels in the center of the main peaks in those histograms are used as seeds for the multiseeded fuzzy connectedness algorithm which groups pixels into homogeneous regions (roughly corresponding to blood, myocardium and lungs). The final histograms are built from these regions.

\section{Segmentation of the First Slice}

The first slice in the dataset (the most basal slice) intersects with the valve plane. This makes it difficult to segment and often, the LV cannot be detected by the first step in Section 2.1. Consequently, we first segment the first slice on which an LV blood pool was detected. It is usually a clean slice, below the valve plane, without many papillary muscles inside the blood pool. It is a very good candidate to start the segmentation process.

\subsection{Deformable Registration}

To segment a slice, we first register all the phases in the slice and generate an average image. To calculate the deformation field between two phases, a variational non-rigid registration algorithm [11] is applied. This approach can be viewed as an extension of the classical optical flow method in which a dense deformation field is estimated as the solution to a calculus of variations problem 


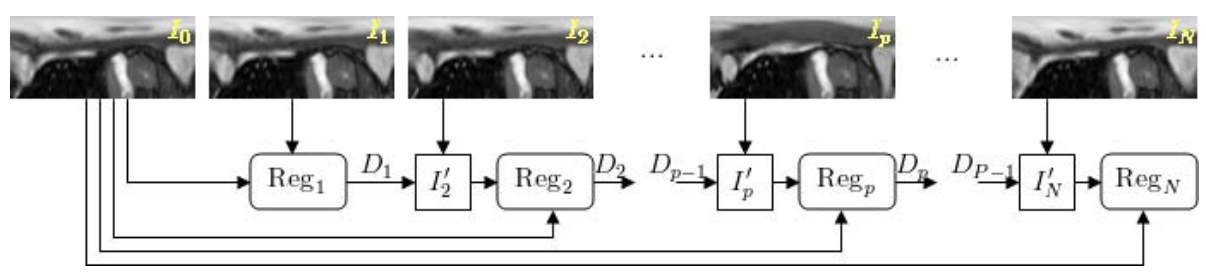

Fig. 2. Strategy to capture the large deformations between pairs of phases (especially $\mathrm{ED}$ and $\mathrm{ES}$ ) during registration in polar space

where the cost function is defined as the sum of an image similarity measure (local cross correlation ratio) and regularization terms.

The registration algorithm is applied to the polar images because the contours will be recovered in polar space and the intrinsic motion of the myocardium is mostly radial [12. To account for the fact that, in polar space, the pixels in the first column of the image are neighbors of the pixels in the last column of the image, we use the tiling boundary condition on the images to calculate derivatives.

Due to the large myocardial contraction during the $\mathrm{R}$ - $\mathrm{R}$ intervals and the finite capture range of non-rigid registration, a simple registration from the ED phase often fails to fully recover the myocardium deformation in other phases (especially the ES phase). We propose the strategy illustrated in Fig. 2 to overcome this limitation. After a phase is selected as the reference image, the next phase is first registered to this reference. Then, the second registration is initialized with the deformation field from the first registration, which is equivalent to first transforming the second phase to the reference coordinate system using a partially accurate deformation field and then running the registration between those to recover the remaining deformation.

\subsection{Segmentation in the Average Temporal Image}

The endocardium and epicardium contours are recovered independently in the average image using a shortest path algorithm for which the main difficulty is to define the cost function. In this case, the strong gradient between the papillary muscles and the blood should be avoided as the contour should stay behind the papillary muscles. Also, the gradient which might be stronger between the lungs and the fat than between the myocardium and the fat is not a good indicator of the epicardium.

Since it is impossible to design the best cost function that will work in all possible cases, we have chosen to use multiple cost functions to recover multiple contour candidates as illustrated in Fig. 3. First, the phases are aligned separately to the ED and ES phases and averaged to produce the ED and ES average images $I_{E D}(x)$ and $I_{E S}(x)$. Then, for each average image, we compute two different myocardium probabilities: a) the distribution probability $\mathcal{M}^{H}(x)$ is the response of the myocardium histogram to the pixels in the average image; $b$ ) 


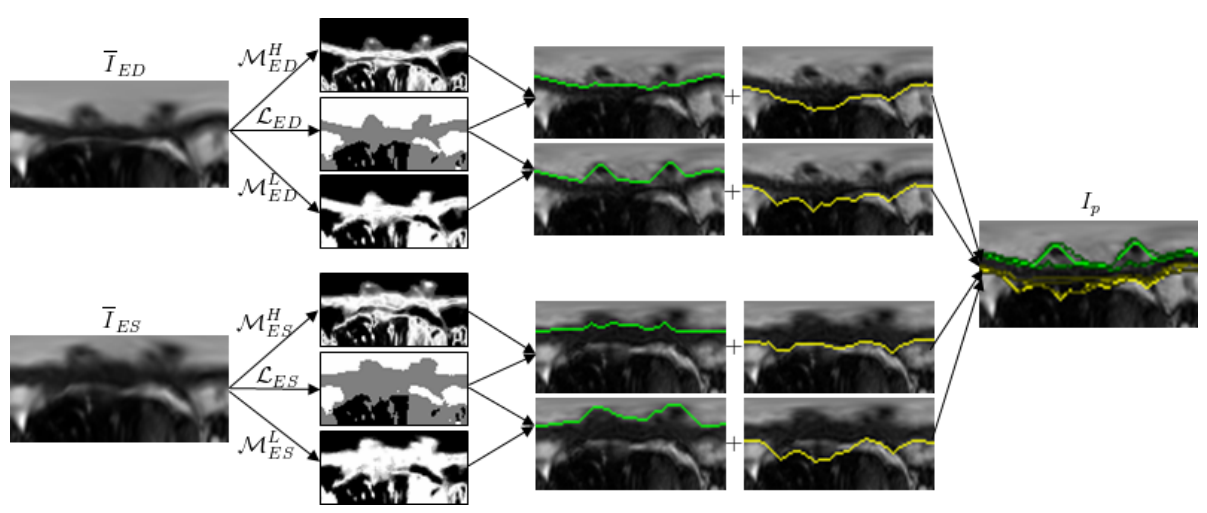

Fig. 3. Segmentation of the average images to generate multiple candidate contours

the label probability $\mathcal{M}^{L}(x)$ is the average of the label images $\mathcal{L}_{p}(x)$ produced by the multiseeded fuzzy connectedness algorithm. Finally, gradient images are computed in the following way. For the endocardium, $g^{\text {endo }}(x)$ is the gradient of $\mathcal{M}(x)$ where gradients in the wrong direction are eliminated because the probability is expected to be bright inside the myocardium. Also pixel transitions in $\mathcal{L}(x)$ from myocardium below to non myocardium above are highlighted. For the epicardium, $g^{\text {epi }}(x)$ is the sum of the gradient of $\mathcal{M}(x)$ and the gradient of $I(x)$. Again, gradients in the wrong direction are eliminated and pixel transitions in $\mathcal{L}(x)$ from blood (lung) below to non blood (non lung) above are highlighted.

The endocardium is recovered first using the gradient cost function defined as $G(x)=\frac{1}{|g(x)|^{2}+\epsilon}$. Then, for the epicardium, three criteria are minimized, namely the gradient cost function, the number of pixels between the two contours not labeled as myocardium in $\mathcal{L}(x)$, and the variation in the thickness of the myocardium. We use Dijkstra's algorithm to recover the shortest path. All the pixels in the leftmost column in the image are initialized as starting points on the path and as soon as a path reaches a pixel in the rightmost column, the algorithm terminates. The contours generated by the shortest path algorithm using the two different probability images for the two average images are transfered back to all the phases using the corresponding deformation fields to obtain four different candidate contours per phase. These contours are then combined using a minimal surface algorithm.

\subsection{Minimal Surface Segmentation of All Phases}

The minimal surface algorithm was developed by Grady [13] to extend the shortest path algorithm to 3D. This extension of the shortest path algorithm accepts one or more closed 2D contours as input and produces the global minimal surface, with respect to the cost function, having these $2 \mathrm{D}$ contours as its boundary.

The cost function is defined as follows for each phase $p$. It is important to follow the edges of the current frame, so the directed gradient images $g_{p}^{\text {endo }}(x)$ and $g_{p}^{\text {epi }}(x)$ of the probability images $\mathcal{M}_{p}^{H}(x)$ and $\mathcal{M}_{p}^{L}(x)$ for the current phase and the gradient cost function $G_{p}(x)=\frac{1}{\left|g_{p}(x)\right|^{2}+\epsilon}$ are computed as described earlier. 


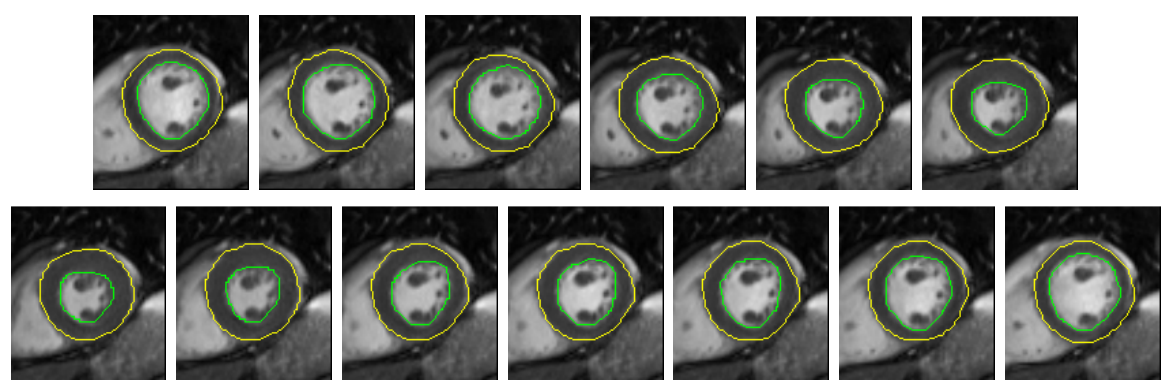

Fig. 4. Segmentation of the first slice (cropped for better viewing)

Then, pixels on the candidate contours are retained based on how likely there are of belonging to the final contour. For the endocardium, since we want the contour to stay behind the papillary muscles, the most promissing pixels are farther from the center of the contour. In polar coordinates, these pixels have a larger row position. Let $i(x)$ be the row position of pixel $x$ and let $i_{1}(x)$ (resp. $\left.i_{2}(x)\right)$ be the row position of the top most (resp. bottom most) contour in the same column as pixel $x$. The likelihood of a pixel on a candidate contour is defined as $F_{p}^{\text {endo }}(x)=\alpha\left(i(x)-i_{1}(x)\right)+\beta$. The situation is more complicated for the epicardium because there are many missing and spurious edges. Once the endocardium has been recovered, we determine the average thickness $T$ of the myocardium using all epicardium candidates in that phase. The likelihood of a pixel on a candidate contour is defined as $F_{p}^{\text {epi }}(x)=\left\|i(x)-\left(i^{\text {endo }}(x)+T\right)\right\|$. In addition, if there are no good edges for the epicardium in a particular area, the pixels $T$ away from the endocardium become good candidate by setting $F_{p}^{\text {epi }}\left(i^{\text {endo }}(x)+T, j\right)=0$. The distance map from these candidate pixels is computed and multiplied to the gradient cost functions to generate the final cost function for the minimal surface algorithm.

To initialize the contours for the minimal surface algorithm, we apply Dijkstra's algorithm in the ED phase to generate a $2 \mathrm{D}$ contour. The $3 \mathrm{D}$ volume consists of all phases with the ED phase as the first phase and ED phase added again as the last phase. This way, the algorithm is initialized with two contours and the minimal surface is generated between them. In order to not bias the algorithm with the initial contour, we apply a second pass where the initial contour is the ES contour from the first pass and the $3 \mathrm{D}$ volume goes from the ES phase to the ES phase. Once the contours have been segmented, they are converted back to Cartesian space. Fig. 4 shows an example of the segmentation in the first slice.

\section{Segmentation of the Other Slices}

The segmentation of the other slices is very similar. The slices are propagated in both directions from the first slice to the apex and to the base. To begin, we apply the deformable registration to align the ED and ES phases of the current 

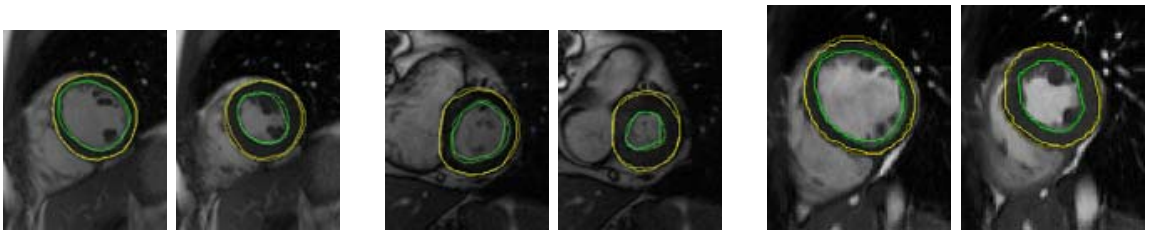

Fig. 5. Segmentation in mid-ventricle slices (cropped for better viewing): automatic contours are shown brighter and ground truth contours are shown dimmer

slice to the previous slice. The contours in the ED and ES phases from the previous slice are transferred to the current slice by applying the deformation field to define shape priors. When the candidate contours are recovered using the shortest path algorithms in the ED and ES average images, the distance maps around the prior contours are combined with the gradient cost functions as follows:

$$
\begin{aligned}
& \hat{G}_{k}^{\text {endo }}(x)=G_{k}^{\text {endo }}(x)\left(D_{k}^{\text {endo }}(x)+1\right) \\
& \hat{G}_{k}^{\text {epi }}(x)=G_{k}^{\text {epi }}(x)\left(D_{k}^{\text {epi }}(x)+1\right)
\end{aligned} \quad k=E D, E S .
$$

Finally, the candidate contours (including the prior contours) are combined using the minimal surface algorithm. Segmentation examples are shown in Fig. 5] where automatic contours are shown in bright while ground truths are dimmer.

\section{Experiments}

To evaluate the performance of the algorithm, we have collected 19 patient datasets from 4 different clinical sites. Two experts manually outlined the endocardium and epicardium in the ED and ES phases for all the datasets. They worked together to produce a single contour set that was agreed upon. We ran our fully automatic algorithm and generated the segmentation contours. The algorithm is quite fast, it takes 1 minute to segment an average dataset with 200 images ( $0.3 \mathrm{~s}$ per image) on a dual core laptop $(2.33 \mathrm{GHz}$ and $2 \mathrm{~GB} \mathrm{RAM})$.

The contours were compared in the following way. To compute the distance between two contours, we first sample the contours so that their vertices are one pixel apart. For each vertex on each contour, we compute the distance to the closest point (not necessarily a vertex) on the other contour. We then plot the cumulative histogram of these distances for all patients (all vertices on all contours) and compute the root mean square (RMS) of these distances. We also compute the average Dice coefficient. Fig. 7 shows the summary of results for all contours, the endocardium and epicardium contours, the most basal slices, the most apical slices, and the mid-ventricular slices. The cumulative histograms are to be read in the following way: a point $(x, y)$ on the curve says that $x \%$ of all distances are below $y$ pixels (pixel sizes for the 19 datasets varied between 1.32 and $2.47 \mathrm{~mm})$. 

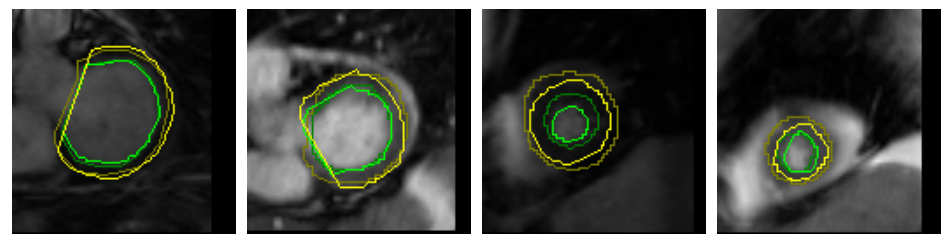

Fig. 6. Segmentation results in the most basal and most apical slices

\begin{tabular}{|l|r|r|r|}
\hline & $\begin{array}{c}\text { RMS } \\
\text { (pixels) }\end{array}$ & $\begin{array}{c}\text { RMS } \\
(\mathrm{mm})\end{array}$ & Dice \\
\hline overall & 1.61 & 2.70 & 0.89 \\
\hline endocardium & 1.48 & 2.48 & 0.88 \\
epicardium & 1.74 & 2.91 & 0.91 \\
\hline basal slices & 1.65 & 2.76 & 0.92 \\
apical slices & 2.36 & 3.92 & 0.78 \\
mid slices & 1.34 & 2.24 & 0.93 \\
\hline \multicolumn{2}{|c|}{ Pixel sizes: $1.32-2.47 \mathrm{~mm}$} \\
\hline
\end{tabular}

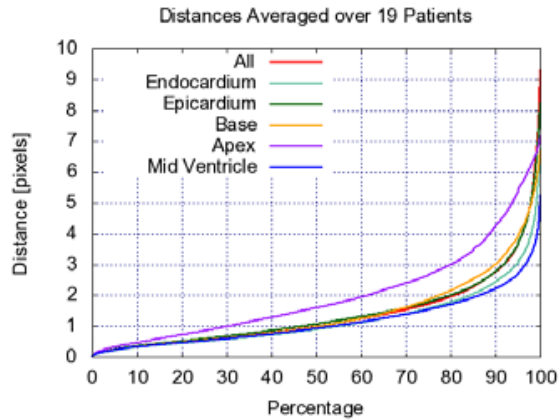

Fig. 7. Distance between ground truth and automatic contours (the RMS distance should be small, the Dice coefficient should be large), a point $(x, y)$ in the graph indicates that $x \%$ of all distances are below $y$

The curves show that the error is around 1 pixel $(\approx 2 \mathrm{~mm})$ half of the time and around 2 pixels $(\approx 4 \mathrm{~mm}) 80 \%$ of the time. There are very few large errors which means that they occur in a few places and can easily be manually edited. We observe that there is not much difference between endocardium and epicardium: the algorithm recovers both contours equally well. The algorithm performs well in the basal slices, but the error is much larger in the apical slices, and it can be seen in the graph in Fig. 7 that the largest $10 \%$ of the distances are significantly lower when those slices are ignored. Actually, very few people in the literature have considered such slices as the ones seen in Fig. 6 where the outflow tract can be seen in the base and the LV blood pool moves in and out of the slice between ED and ES (no ground truth was defined on the ES phases of those slices) and where the endocardium is very small in the apical slices.

\section{Conclusions}

We have proposed a fully automatic system to segment the left ventricle myocardium from cine MR images. The method combines deformable registration, shortest paths, and minimal surfaces. We demonstrated on 19 datasets that the results look very good and the errors are small enough that the system can be used in clinical settings. In the future, we would like to combine this short axis segmentation with a long axis segmentation (similar to [4) as it will help in resolving the more difficult cases in the basal and apical slices. 


\section{References}

1. Lorenzo-Valdés, M., Sanchez-Ortiz, G., Elkington, A., Mohiaddin, R., Rueckert, D.: Segmentation of $4 \mathrm{D}$ cardiac MR images using a probabilistic atlas and the EM algorithm. Medical Image Analysis 8 (2004)

2. Jolly, M.: Automatic segmentation of the left ventricle in cardiac MR and CT images. International Journal of Computer Vision 70(2) (2006)

3. Mitchell, S., Lelieveldt, B., van der Geest, R., Bosch, H., Reiber, J., Sonka, M.: Multistage hybrid active appearance model matching: Segmentation of the left and right ventricles in cardiac MR images. IEEE Trans. Medical Imaging 20(5) (2001)

4. Fradkin, M., Ciofolo, C., Mory, B., Hautvast, G., Breeuwer, M.: Comprehensive segmentation of cine cardiac MR images. In: Metaxas, D., Axel, L., Fichtinger, G., Székely, G. (eds.) MICCAI 2008, Part I. LNCS, vol. 5241, pp. 178-185. Springer, Heidelberg (2008)

5. Lynch, M., Ghita, O., Whelan, P.F.: Segmentation of the left ventricle of the heart in 3-D+t MRI data using an optimized nonrigid temporal model. IEEE Trans. Medical Imaging 27(2) (2008)

6. Sun, W., Çetin, M., Chan, R., Willsky, A.: Segmentation of the evolving left ventricle by learning the dynamics. In: ISBI (2008)

7. Paragios, N.: A level set approach for shape-driven segmentation and tracking of the left ventricle. IEEE Trans. Medical Imaging 22(6) (2003)

8. Lorenzo-Valdés, M., Sanchez-Ortiz, G.I., Mohiaddin, R.H., Rueckert, D.: Atlasbased segmentation and tracking of 3D cardiac MR images using non-rigid registration. In: Dohi, T., Kikinis, R. (eds.) MICCAI 2002. LNCS, vol. 2488, p. 642. Springer, Heidelberg (2002)

9. Jolly, M.-P.: Automatic recovery of the left ventricular blood pool in cardiac cine MR images. In: Metaxas, D., Axel, L., Fichtinger, G., Székely, G. (eds.) MICCAI 2008, Part I. LNCS, vol. 5241, pp. 110-118. Springer, Heidelberg (2008)

10. Jolly, M.P., Grady, L.: 3D general lesion segmentation in CT. In: ISBI (2008)

11. Hermosillo, G., Chefd'hotel, C., Faugeras, O.: Variational methods for multimodal image matching. International Journal of Computer Vision 50(3) (2002)

12. Noble, N., Hill, D., Breeuwer, M., Schnabel, J., Hawkes, D., Gerritsen, F., Razavi, R.: Myocardial delineation via registration in a polar coordinate system. In: Dohi, T., Kikinis, R. (eds.) MICCAI 2002. LNCS, vol. 2488, p. 651. Springer, Heidelberg (2002)

13. Grady, L.: Computing extact discrete minimal surfaces: Extending and solving the shortest path problem in 3D with application to segmentation. In: CVPR (2006) 